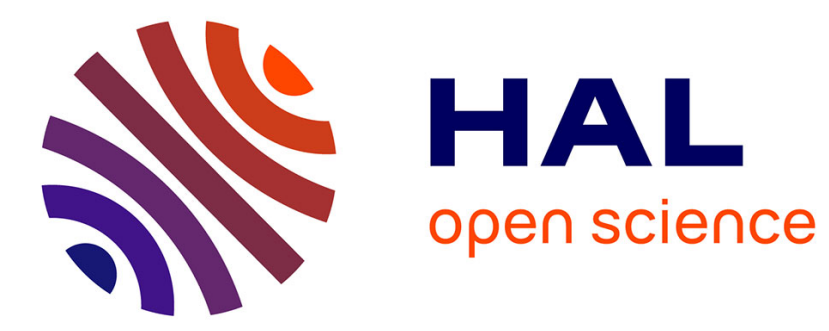

\title{
Bifurcations in the wake of a thick circular disk
}

Franck Auguste, David Fabre, Jacques Magnaudet

\section{To cite this version:}

Franck Auguste, David Fabre, Jacques Magnaudet. Bifurcations in the wake of a thick circular disk. Theoretical and Computational Fluid Dynamics, 2010, vol. 24, pp. 305-313. 10.1007/s00162-0090144-3 . hal-00877297

\section{HAL Id: hal-00877297 https://hal.science/hal-00877297}

Submitted on 28 Oct 2013

HAL is a multi-disciplinary open access archive for the deposit and dissemination of scientific research documents, whether they are published or not. The documents may come from teaching and research institutions in France or abroad, or from public or private research centers.
L'archive ouverte pluridisciplinaire HAL, est destinée au dépôt et à la diffusion de documents scientifiques de niveau recherche, publiés ou non, émanant des établissements d'enseignement et de recherche français ou étrangers, des laboratoires publics ou privés. 


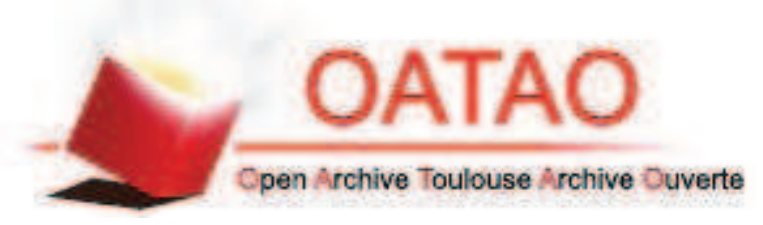

\section{Open Archive TOULOUSE Archive Ouverte (OATAO)}

OATAO is an open access repository that collects the work of Toulouse researchers and makes it freely available over the web where possible.

This is an author-deposited version published in : http://oatao.univ-toulouse.fr/ Eprints ID : 9797

To link to this article : DOI: $10.1007 / \mathrm{s} 00162-009-0144-3$

URL : http://dx.doi.org/10.1007/s00162-009-0144-3

To cite this version Auguste, Franck and Fabre, David and Magnaudet, Jacques Bifurcations in the wake of a thick circular disk. (2010) Theoretical and Computational Fluid Dynamics, vol. 24 (n 1-4). pp. 305-313. ISSN 0935-4964

Any correspondance concerning this service should be sent to the repository administrator: staff-oatao@,1istes-diff.inp-toulouse.fr 


\title{
Franck Auguste · David Fabre • Jacques Magnaudet Bifurcations in the wake of a thick circular disk
}

\begin{abstract}
Using DNS, we investigate the dynamics in the wake of a circular disk of aspect ratio $\chi=d / w=3$ (where $d$ is the diameter and $w$ the thickness) embedded in a uniform flow of magnitude $U_{0}$ perpendicular to its symmetry axis. As the Reynolds number $R e=U_{0} d / v$ is increased, the flow is shown to experience an original series of bifurcations leading to chaos. The range $R e \in[150,218]$ is analysed in detail. In this range, five different non-axisymmetric regimes are successively encountered, including states similar to those previously identified in the flow past a sphere or an infinitely thin disk, as well as a new regime characterised by the presence of two distinct frequencies. A theoretical model based on the theory of mode interaction with symmetries, previously introduced to explain the bifurcations in the flow past a sphere or an infinitely thin disk (Fabre et al. in Phys Fluids 20:051702, 2008), is shown to explain correctly all these results. Higher values of the Reynolds number, up to 270 , are also considered. Results indicate that the flow encounters at least four additional bifurcations before reaching a chaotic state.
\end{abstract}

Keywords Wake instabilities · Bifurcation theory

PACS 47.15. Fe Stability of laminar flows, 47.15. Tr Laminar wakes, 47.10. Fg Dynamical systems methods

\section{Introduction}

Bodies moving within a viscous fluid may display a large variety of dynamical behaviours. A famous example, described five centuries ago by Leonardo da Vinci is the rise of small bubbles which may follow zigzagging or spiraling motions [13]. Another example, which puzzled Maxwell since 1857, is the falling of a paper card, which may display various motions such as fluttering, tumbling, chaotic oscillations, etc. [15, 17]. It is now recognized [10] that such path instabilities are directly linked to an instability of the recirculating region in the near wake of the body. Therefore, to understand these movements it is useful to consider first the related and simpler problem of bodies held fixed within a uniform incoming flow.

In a recent paper [5], we used DNS to investigate the bifurcation scenarios in the wake of two reference bodies, namely a sphere and an infinitely thin disk held normal to the stream. For both bodies the Reynolds number is defined as $R e=\frac{U_{0} d}{v}$, where $U_{0}$ is the incoming velocity, $d$ is the diameter of the body, and $v$ is the kinematic viscosity of the fluid. The case of the sphere is well known from the literature $[3,9,12,14,16]$. A first bifurcation occurs for $R e \approx 210$ resulting in a wake characterized by the presence of a steady pair of streamwise vortices and the occurrence of a constant lift force on the body. This wake configuration, hereinafter

F. Auguste $\cdot$ D. Fabre $(\bowtie) \cdot J$. Magnaudet

Institut de Mécanique des Fluides de Toulouse, University of Toulouse, Toulouse, France

E-mail: auguste@imft.fr

E-mail: david.fabre@imft.fr; fabred@imft.fr

E-mail: magnau@imft.fr 
referred to as the "Steady-State" $(S S)$ mode, breaks the axisymmetry but retains a reflectional symmetry with respect to an azimuthal plane. A second bifurcation is observed for $R e \approx 272$, leading to a periodic state which retains the symmetry plane and is associated with a lift force oscillating around a non-zero mean value. In experiments, this mode leads to the shedding of hairpin vortices all oriented in the same direction [14]; hence we will refer to it as the "Zig-Zig" mode $(Z z$, to be distinguished with the "Zig-Zag" mode $(Z Z)$ which will be introduced below).

In the case of a flat disk, a different bifurcation sequence is observed. A first bifurcation is observed for $R e=115.5$, leading to a $S S$ mode with a reflectional symmetry. Then a Hopf bifurcation is found for $R e=121.5$. Unlike the case of the sphere, the mode observed after this bifurcation does not preserve the reflectional symmetry. The resulting mode is characterized by a lift force oscillating in a direction around a mean orientation; it can be described as a "Yin-Yang" $(Y Y)$ mode, owing to the characteristic pattern observed just behind the body (see Fig. 4 of the present paper). A third bifurcation is observed for $\operatorname{Re}=139.4$, leading to a recovery of the planar symmetry. The resulting flow is characterized by a lift force oscillating along a given direction with a zero mean. This mode is referred to as a "Standing-Wave" $(S W)$ mode by analogy with other related problems. It can also be called the "Zig-Zag" $(Z Z)$ mode, as in experiments it would be associated with the shedding of hairpin vortices in alternating directions. To explain these results, Fabre et al. [5] introduced a system of nonlinear amplitude equations describing the interaction between the two dominant unstable modes. This model allowed us to explain the differences observed in the wake of the two bodies and accurately reproduces the evolution of the lift forces. For the case of an infinitely thin disk, these conclusions were recently confirmed by Meliga et al. [11], who obtained the same system of equations using a weakly nonlinear global stability approach.

In the present paper, we continue the numerical exploration of the wake dynamics of axisymmetric bodies by considering a geometry which is somehow intermediate between that of a sphere and that of a thin disk, namely a thick disk with an aspect ratio $\chi=d / w=3$, where $d$ is the diameter and $w$ the thickness. This geometry was chosen because an extensive experimental study was carried out in our team with freely moving bodies of this type rising in salted water [6,7]. In Sect. 2 we describe the numerical method we use to investigate the flow past this body. In Sect. 3 we describe the various flow regimes we observe as the Reynolds number is increased in the range $R e \in[150,218]$. In Sect. 4 we show that the theoretical model introduced by Fabre et al. [5] can be adapted to explain these computational results. In Sect. 3, we briefly address the transition to chaos occurring in the range $R e \in[218,270]$. We finally provide some conclusions in Sect. 6.

\section{Numerical method and strategy of investigation}

The numerical code used in the present study is similar to that used in [5] and was described in [1,10]. The code solves the three-dimensional Navier-Stokes equations for an incompressible and homogeneous fluid. Temporal evolution is discretized by a third-order Runge-Kutta scheme. The divergence-free condition is satisfied using a projection method. The flow is described using a cylindrical grid with $118(x) \times 70(r) \times 32(\theta)$ nodes (the $x$-axis corresponds to the symmetry axis). A nonuniform grid distribution is used near the body to properly capture the boundary layer and near wake. The characteristic grid size is $0.015 d$ near the body, and is about $0.1 d$ in the near wake (down to $x=2 d$ ). The grid extends to a distance of roughly 10 diameters in all directions. The boundary conditions are (i) a no-slip condition on the body itself, (ii) a kinematic condition $u_{x}=U_{0}$ in the inlet plane and on the lateral boundaries, and (iii) a non-reflecting outlet condition in the outlet plane. The grid used here provides results in good agreement with those found during the convergence study performed for a thin disk by Auguste et al. [1].

The range of Reynolds numbers $R e \in[150,220]$ was scrutinized with steps of $\Delta R e=1$ in most of the interval, and not larger than $\Delta R e=2$. Computations were run on the Altix server computer of CICT in Toulouse (parallelized on two processors). Typical runs consisted of 50000 time steps (corresponding to a dimensionless time $t U_{0} / d$ about 800 ); however, much longer computational times were required to achieve convergence close to some of the bifurcations. Typically, computations were started with an initial velocity field originating from a former computation performed with a neighbouring value of $R e$; close to the bifurcations, the Reynolds number was varied both upwards and downwards to detect an eventual subcritical behaviour. Most of the bifurcations described here were localized within an interval $\Delta R e=1$; however, in some cases, observing the transient behavior and fitting using theoretical results, as done for instance in [3], allowed a more accurate estimate of the threshold values. 
To describe the efforts experienced by the body, we introduce the drag coefficient $C_{x}$, the side force coefficients $C_{y}$ and $C_{z}$, and the torque coefficients $C_{m x}, C_{m y}$ and $C_{m z}$ defined as follows:

$$
\begin{aligned}
\left(F_{x}, F_{y}, F_{z}\right) & =\left(C_{x}, C_{y}, C_{z}\right) \rho U_{0}^{2} \pi(d / 2)^{2} / 2 . \\
\left(M_{x}, M_{y}, M_{z}\right) & =\left(C_{m x}, C_{m y}, C_{m z}\right) \rho U_{0}^{2} \pi(d / 2)^{3}
\end{aligned}
$$

To describe the various flow regimes, we draw two types of "phase diagrams", based respectively on side forces $\left(C_{y}-C_{z}\right.$ diagram) and drag/lift forces $\left(C_{x}-C_{L}\right.$ diagram, where $C_{L}=\sqrt{C_{y}^{2}+C_{z}^{2}}$ is the lift coefficient).

Finally, note that the exact or average reflectional symmetry planes existing in some of the regimes described in the next section are arbitrary and are selected by the initial conditions of the simulation. To make the discussion simpler, a convenient rotation was applied to the numerical results to make the symmetry directions coincide with the $y$ - or $z$-axis.

\section{The sequence of bifurcations in the range $R e \in[150,217]$}

As for the reference cases of a sphere and a flat disk, the flow remains steady and axisymmetric at low enough values of the Reynolds number. In this range, the flow is characterised by a toroidal recirculation region (except at very low values of $R e$ where the latter vanishes). Such a flow, hereinafter referred to as the "trivial" state $(T S)$ is illustrated in Fig. 1a, which displays the vorticity and streamlines in a transverse plane for $R e=150$. In this regime the hydrodynamic force on the body reduces to a drag force.

As the Reynolds number increases, a steady bifurcation occurs. In agreement with the reference cases of a sphere and a disk, it leads to a "Steady-State" mode $(S S)$ characterised by a reflectional symmetry plane and an associated lift force. The threshold value associated with this bifurcation was estimated to $R e_{c 1} \approx 159.4$, in agreement with the previous experimental estimate $R e_{c 1}=116.5\left(1+\chi^{-1}\right)$ proposed by Fernandes et al. [6]. The flow in this regime is illustrated in Fig. 1b which displays iso-surfaces of the streamwise vorticity component (through two orthogonal views). In this case, the symmetry plane is the $x-z$ plane and the lift force is along the positive $z$ direction.

The next bifurcation is of Hopf type and occurs for a threshold Reynolds number estimated to $\operatorname{Re}_{c 2} \approx 179.8$. This value also reasonably matches the previous estimate $R e_{c 2}=125.6\left(1+\chi^{-1}\right)$ [6]. Above this threshold, the flow within the wake and the forces experienced by the body become unsteady and periodic. A Strouhal number characterising this flow can be defined as $S t=\frac{d}{U_{0} T_{0}}$, where $T_{0}$ is the shedding period. Right at the threshold, this Strouhal number is $S t=0.109$, a value comparable to but slightly smaller than those found for the two reference bodies $\left(S t_{\text {sphere }} \approx 0.133, S t_{\text {disk }} \approx 0.119\right)$. The regime resulting from this bifurcation is illustrated in Fig. 2. It can be observed that the wake retains the symmetry plane selected by the previous bifurcation. This allows us to identify this mode as the "Zig-zig" one already observed in the wake of a sphere. Figure $2 \mathrm{a}$ shows the structure of the flow at two instants of the shedding cycle. The $C_{y}-C_{z}$ diagram (Fig. 2b) indicates that the lift force is oscillating about a non-zero mean value along a line located within the symmetry plane of the flow. The $C_{x}-C_{L}$ diagram (Fig. 2c) reveals a single loop. This loop travels in the anti-clockwise

(a)

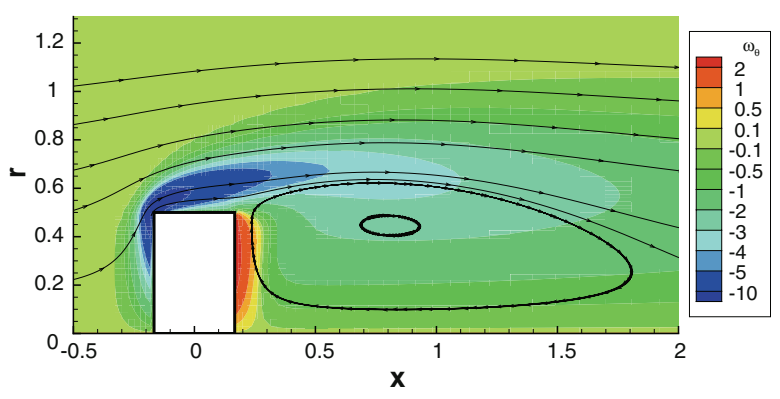

(b)

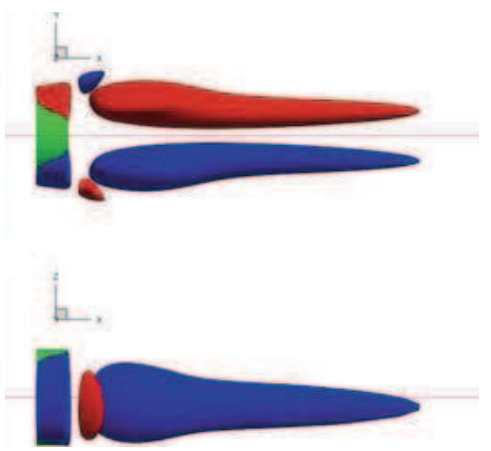

Fig. 1 Steady flow regimes: a axisymmetric flow for $R e=150$ depicted by azimuthal vorticity (colors online) and streamlines, b non-axisymmetric steady state $(S S)$ for $R e=165$ depicted by iso-surfaces of the streamwise vorticity (two orthogonal views) 
(a)

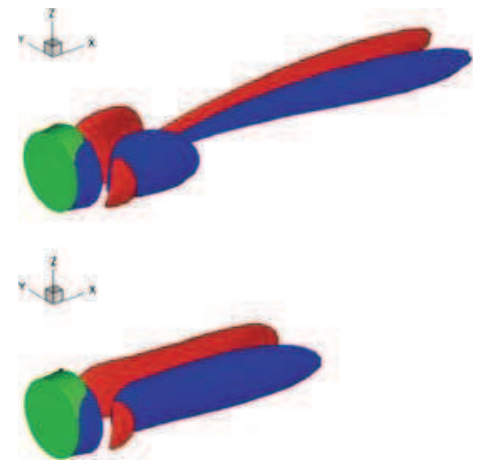

(b)

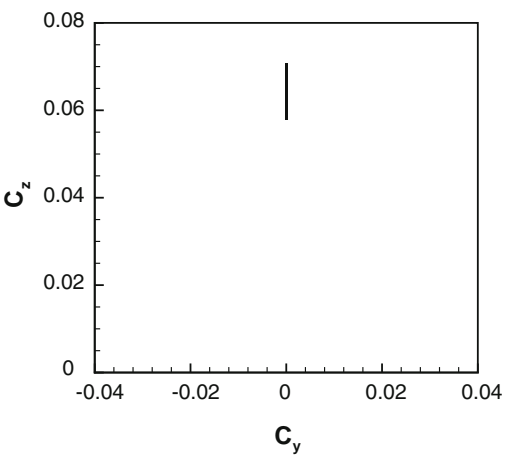

(c)

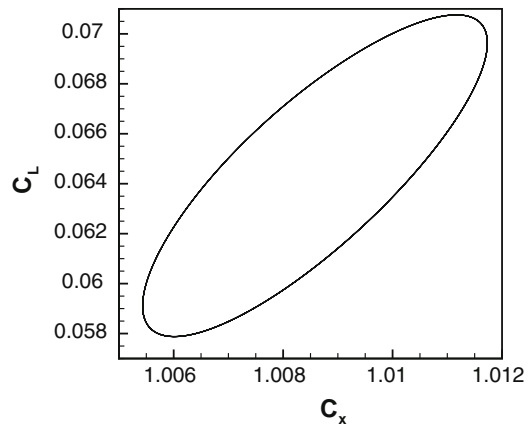

Fig. 2 Periodic, reflectional-symmetry-preserving mode (or "Zig-Zig" mode) for $R e=182$. a Iso-surfaces of streamwise vorticity (at two time instants of the cycle corresponding to the maximum and the minimum of the lift force, respectively), $\mathbf{b} C_{y}-C_{z}$ diagram, $\mathbf{c} C_{x}-C_{L}$ diagram

(a)

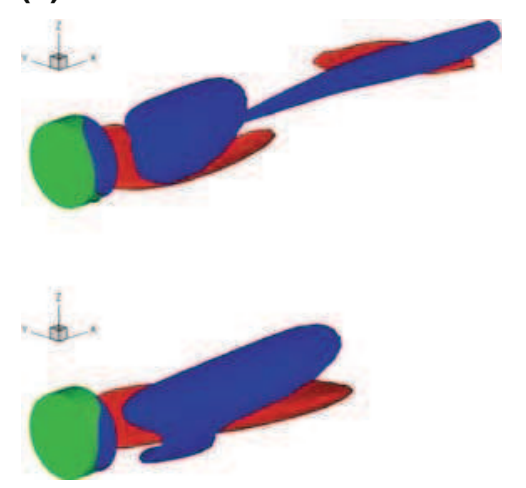

(b)

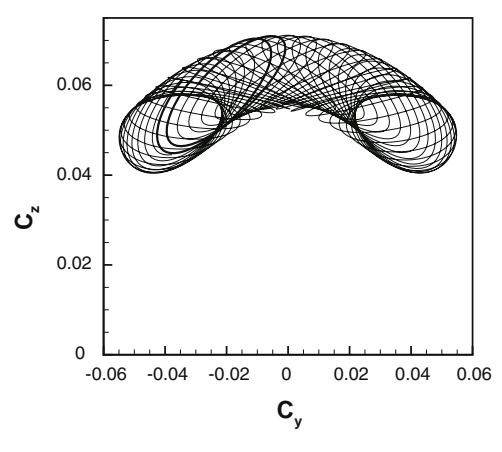

(c)

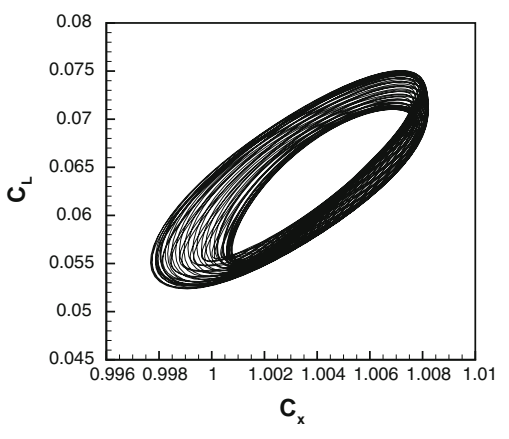

Fig. 3 Quasi-periodic pulsating mode (or "Knit-Knot" mode) for $R e=187$. a Iso-surfaces of the streamwise vorticity at two instants of time, b $C_{y}-C_{z}$ diagram (the end of the time series is represented with a thick line), $\mathbf{c} C_{x}-C_{L}$ diagram

direction, indicating that extrema in the drag force are encountered slightly before those of the lift force. Note that the Strouhal number characterising this flow regime (and actually also the three next regimes) is remarkably constant, and only varies by a few percents around the value $S t=0.109$ selected at $R e=R e_{c 2}$.

A third bifurcation is found to take place for a threshold value $R e_{c 3} \in[184,185]$. The flow observed beyond this bifurcation is illustrated in Fig. 3 (for $R e=187$ ). This flow is of a totally new type, and is characterised by the breaking of the reflectional symmetry (as can be seen in Fig. 3a which shows the structure of the streamwise vorticity at two sample instants) and the occurrence of a secondary frequency. The $C_{y}-C_{z}$ diagram (Fig. 3b) reveals an attractor with a complicated structure evoking the shape of a wool ball. This is why we term this regime the "Knit-Knot mode" $(K K)$. The motion along the attractor can be understood as the superposition of two movements, namely a rapid motion along an elliptic path, with a period $T_{0}$ very close to that found in the previous regime, and a slow pulsation of this ellipse around a mean direction (which corresponds to the $z$ direction in the figure). To help understand this motion, the last two elliptic oscillations are displayed with thick lines in the figure. The $C_{x}-C_{L}$ diagram shows another projection of the attractor which also reveals a two-period motion. In this diagram the pulsation is seen as a slight jitter of the main cycle. Note that the oscillation period $T_{p}$ greatly varies in the range of existence of this mode, from $T_{p} \approx 96 T_{0}$ at $\operatorname{Re}=185$, down to $T_{p} \approx 48 T_{0}$ at $\operatorname{Re}=187$, and then up to $T_{p} \approx 54 T_{0}$ at $\operatorname{Re}=190$. On the other hand the main period remains remarkably constant.

The "Knit-knot" mode described above is observed up to a threshold value of $\operatorname{Re}_{c 4} \in[190,191]$ where a fourth bifurcation is detected. This bifurcation is characterised by the disappearance of the above slow pulsation, and the flow then comes back to a purely periodic state. The resulting flow regime is illustrated in Fig. 4 (for $R e=195$ ). The structure of the streamwise vorticity (Fig. 4a) allows us to identify this mode with the mode existing in the wake of a thin disk in the range $R e \in[121,139]$ [5]. The $C_{y}-C_{z}$ diagram (Fig. 4b) 
(a)

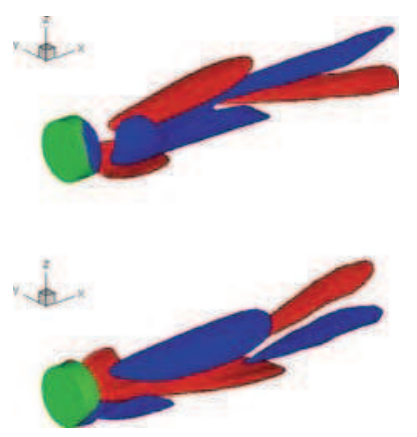

(b)

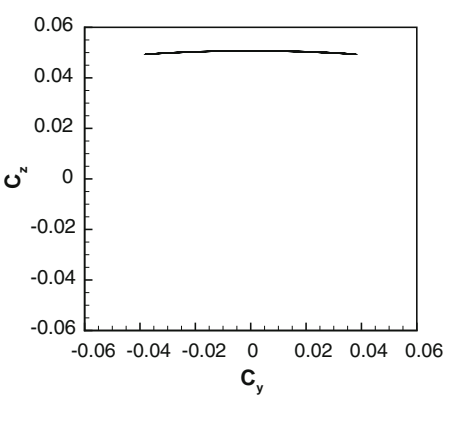

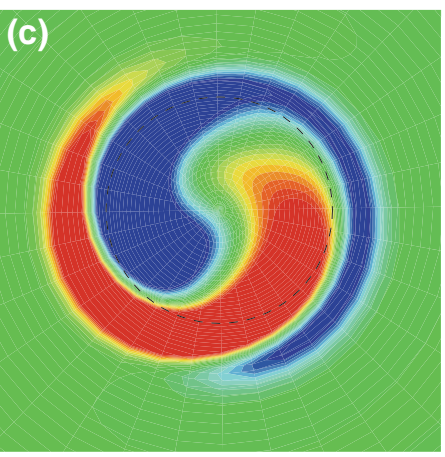

Fig. 4 Periodic, reflectional-symmetry-breaking mode (or "Yin-Yang" mode) for $R e=195$. a Iso-surfaces of the streamwise vorticity at two instants, $\mathbf{b} C_{y}-C_{z}$ diagram, $\mathbf{c}$ iso-levels of the streamw vorticity in the $x=0.5$ plane

(a)

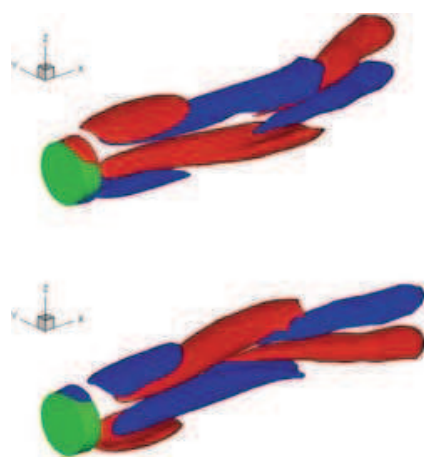

(b)

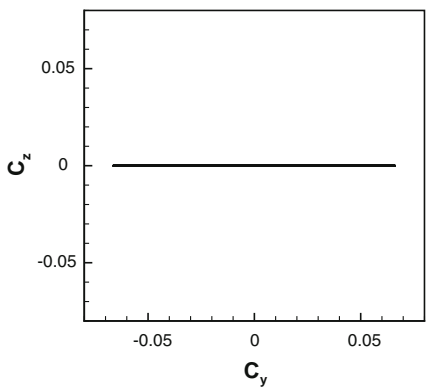

(c)

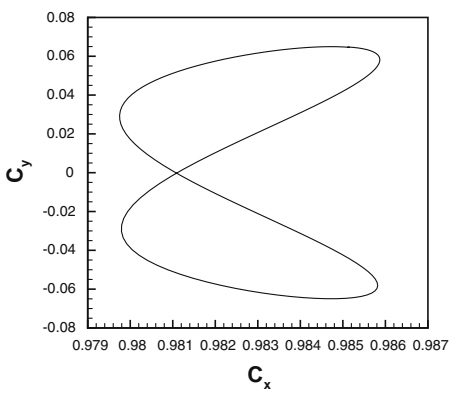

Fig. 5 Periodic mode with reflectional symmetry and zero mean lift (or "Zig-Zag" mode) for $R e=216$. a Iso-levels of the streamwise vorticity in the plane located at $x=0.5, \mathbf{b} C_{y}-C_{z}$ diagram, $\mathbf{c} C_{x}-C_{L}$ diagram

shows that the lift force is oscillating back and forth along a closed path about a mean direction (here chosen to be the $z$ direction), while the $C_{x}-C_{L}$ diagram (not shown) reveals a single loop. This mode was called the "reflectional-symmetry-breaking" $(R S B)$ mode in [5]. It can also be termed as the "Yin-Yang mode" owing to the characteristic shape of the streamwise vorticity contours in a cross-section of the wake (see Fig. 4c).

The next (i.e. fifth) bifurcation is found for $R e_{c 5} \approx 215$. This bifurcation is in all respects similar to that occurring in the wake of a thin disk for $R e \approx 139$, as it is associated with a recovery of a symmetry plane in the wake, the orientation of which (here $y$ ) is orthogonal to that selected by the initial bifurcation (here $z$ ). The resulting flow is illustrated in Fig. 5. The $C_{y}-C_{z}$ diagram (Fig. 5b) indicates that the lift force is contained within the symmetry plane of the flow and is oscillating around a zero mean value. The $C_{x}-C_{y}$ drag-lift diagram (Fig. 5c) shows a butterfly-like attractor, revealing that the drag passes twice through a maximum (and then a minimum) during one period of oscillation of the lift force. The structure of the flow is illustrated in Fig. 5a at two instants of time corresponding, respectively, to a positive extremum (upper plot) and a negative extremum (lower plot) of $C_{y}$. The plots show that in this case the body sheds symmetrical hairpin-like structures during each half-period of oscillation, thus justifying the denomination of this regime as a "Zig-Zag mode" $(Z Z)$.

A synthetic view of all the regimes described so far is given in Fig. 6 which presents the maximum and minimum values of force coefficients (Fig. 6a) and torque coefficients (Fig. 6b) as a function of the Reynolds number in the range $R e \in[150,220]$. As can be noticed, all quantities are continuous across the bifurcations (but generally have discontinuous slopes), indicating that all bifurcations are regular and supercritical. The first bifurcation at $R e_{c 1}$ is associated with the onset of a lift force and a lateral torque and also with an increase of the drag force compared to that associated with the axisymmetric solution (displayed with a dotted line in Fig. 6a). Such a discontinuity in the slope of the $C_{x}$ curve has also been reported in the case of a sphere [3]. At the second bifurcation (i.e. for $R e=R e_{c 2}$ ), the lift and drag forces and the side torque become unsteady 

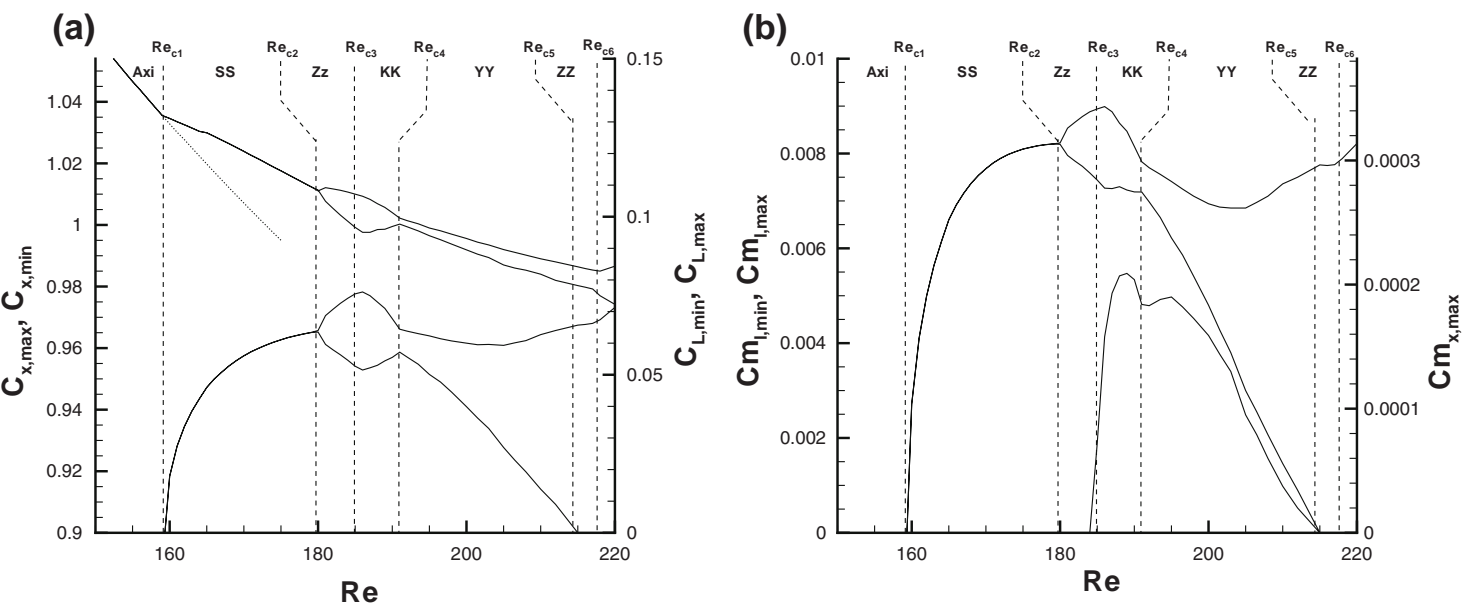

Fig. 6 Variations of force coefficients (a) and torque coefficients (b) in the range $R e \in[150,220]$

and oscillate between the maximum and minimum values plotted in the figure. The third bifurcation at $R e_{c 3}$ is characterised by the occurrence of an axial torque which oscillates about zero with a maximum amplitude represented by the $C_{m, x}$ curve. The presence of such an axial torque is characteristic of regimes in which the reflectional symmetry is broken. Consequently, this torque is non-zero within the range of existence of the "Knit-knot" and "Ying-yang" modes and dies out at the fifth bifurcation corresponding to $R e=R e_{c 5}$.

\section{Theoretical modelling}

In a previous study [5], we introduced a theoretical model which successfully reproduces the first steps of the bifurcation sequence observed in the cases of a sphere and a thin disk. In this section we review this model and show how it can be adapted to the present case.

The model is based on a velocity field with the following expansion:

$$
\mathbf{u}=\mathbf{U}_{0}(r, x)+\operatorname{Re}\left[a_{0}(t) e^{-i \theta} \hat{\mathbf{u}}_{s}(r, x)\right]+\operatorname{Re}\left[a_{1}(t) e^{-i \theta} \hat{\mathbf{u}}_{h,-1}(r, x)+a_{2}(t) e^{i \theta} \hat{\mathbf{u}}_{h,+1}(r, x)\right]+\cdots,
$$

where $\mathbf{U}_{0}(r, x)$ denotes the axisymmetric solution of the Navier-Stokes equations for a given value of $R e$, $\hat{\mathrm{u}}_{s}(r, x)$ is the most amplified mode (associated with an azimuthal wavenumber $m=1$ and a real eigenvalue $\lambda_{s}$ ), and $\hat{\mathrm{u}}_{h, m}(r, x)$ is the next most amplified mode (associated with an azimuthal wavenumber $m=1$ and a complex eigenvalue $\lambda_{h}+i \omega_{h}$ ), whereas $a_{0}, a_{1}, a_{2}$ are three complex amplitudes.

Starting from expansion Eq. (1), the central manifold theorem states that if the leading modes are simultaneously nearly neutral, the whole problem can be reduced to a system of ordinary differential equations (ODE) governing their amplitudes $[4,8]$. This ODE system has the generic form, known as the normal form of the problem:

$$
\begin{aligned}
& \dot{a_{0}}=\lambda_{s} a_{0}+l_{0}\left|a_{0}\right|^{2} a_{0}+l_{1}\left(\left|a_{1}\right|^{2}+\left|a_{2}\right|^{2}\right) a_{0}+i l_{2}\left(\left|a_{2}\right|^{2}-\left|a_{1}\right|^{2}\right) a_{0}+l_{3} \overline{a_{0}} \overline{a_{2}} a_{1}, \\
& \dot{a_{1}}=\left(\lambda_{h}+i \omega_{h}\right) a_{1}+\left(B\left|a_{1}\right|^{2}+(A+B)\left|a_{2}\right|^{2}\right) a_{1}+C\left|a_{0}\right|^{2} a_{1}+D a_{0}^{2} a_{2}, \\
& \dot{a_{2}}=\left(\lambda_{h}+i \omega_{h}\right) a_{2}+\left(B\left|a_{2}\right|^{2}+(A+B)\left|a_{1}\right|^{2}\right) a_{2}+C\left|a_{0}\right|^{2} a_{2}+D \overline{a_{0}} a_{1},
\end{aligned}
$$

where $l_{0}$ to $l_{3}$ are real coefficients, while $A, B, C, D$ are complex. Interestingly, this system is also relevant to the Taylor-Couette flow problem where it describes the interaction between "Taylor vortices" and "spiral vortices". A classification of the solutions up to secondary bifurcations is available in [8]. A refined investigation of the possible solutions up to ternary bifurcations, and of their relevance to the present problem, is in progress (Fabre and Knobloch, in preparation).

In Ref. [5] the coefficients involved in (4) were fitted using the computational results obtained for the sphere and the thin disk, leading to a good agreement between the predictions of the model and the DNS 


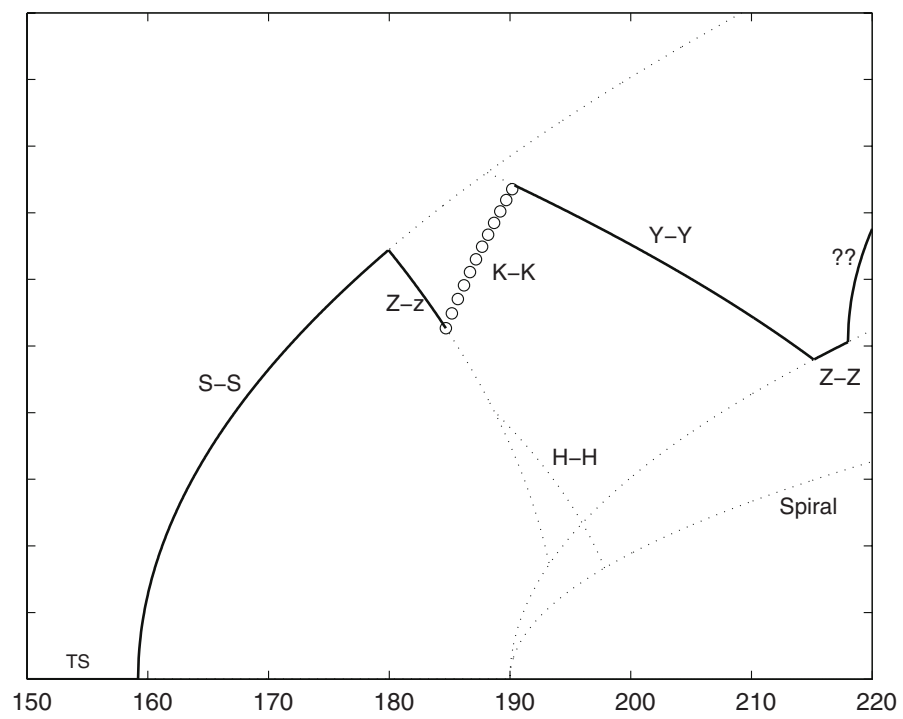

Fig. 7 Theoretical bifurcation diagram. Arbitrary bifurcation parameter as function of the Reynolds number. Full (resp. dashed) lines represent stable (resp. unstable) branches; circles represent the quasi-periodic pulsating "Knit-Knot" branch

results. We have repeated this approach in the present case. The corresponding fitting led to the following set of coefficients:

$$
\begin{aligned}
\lambda_{s} & =0.05(R e-159.8), \quad \lambda_{h}=0.04(R e-190), \quad \omega_{h}=0.685, \quad l 0=-100, \quad l_{1}=-1386, \quad l_{2}=0, \\
l_{3} & =-1800, \quad A=100, \quad B=-225, \quad C=22, \quad D=16.8+50 i .
\end{aligned}
$$

This set of parameters leads to a sequence of bifurcations in close agreement with our numerical results. In particular, the predicted threshold Reynolds number values are as follows:

$$
R e_{c 1}=159.8, \quad R e_{c 2}=179.9, \quad R e_{c 3}=184.7, \quad R e_{c 4}=190.4, \quad R e_{c 5}=215.2 .
$$

The theoretical bifurcation diagram obtained with this set of parameters is plotted in Fig. 7. The figure displays an arbitrary measure of the amplitude of the different states (roughly proportional to $\sqrt{\left|a_{0}\right|^{2}+\left|a_{1}\right|^{2}+\left|a_{2}\right|^{2}}$ ) as function of the Reynolds number; following the usual convention, full (resp. dashed) lines correspond to stable (resp. unstable) branches and circles to quasi-periodic solutions. This diagram correctly reproduces the sequence of bifurcations revealed by the numerical simulations up to $R e=217$, and accurately predicts the threshold values of the successive bifurcations, thus confirming the relevance of the model. Note that it also predicts the existence of two additional branches, namely a "spiral" mode and a quasi-periodic mode of a different nature (termed $H-H$ in the figure), which are both unstable and thus not numerically observed.

A more quantitative fitting of the lift forces, as performed in Ref. [5] for the thin disk, was not tried. Therefore, the set of coefficients given above is only indicative. A direct and rigorous determination of the coefficients of the normal form using a weakly nonlinear expansion of the Navier-Stokes equations, as done by Meliga et al. [11] in the case of a thin disk, would certainly be preferable.

We finally point out that the present model is unable to account for the subsequent bifurcations to be described in the next section, which are most likely associated with the emergence of new leading modes, in addition to the two modes already included in the expansion (1).

\section{The route to chaos in the range $R e \in[217,270]$}

We now turn to the next features that occur at higher Reynolds number. To this end, we investigated the range of Reynolds numbers $[217,270]$ in a less exhaustive way than reported in the previous section. Figure 8 displays sample phase diagrams of the regimes we detected.

The "Zig-Zag" mode, which was the last of the sequence of bifurcations documented in Sect. 3, has a limited range of existence, as a new bifurcation occurs for $R e_{c 6} \in[217,218]$. The mode observed beyond this 
(a)

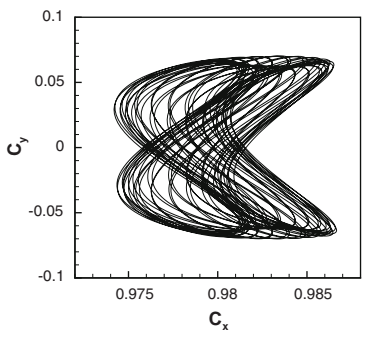

(b)

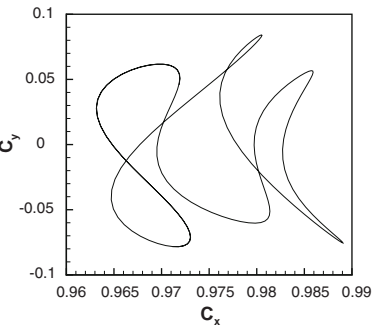

(c)

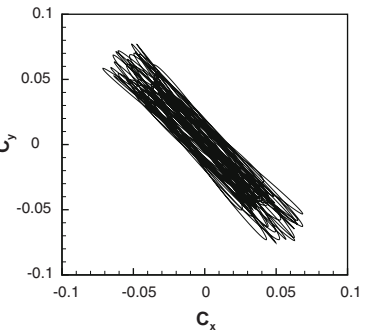

(d)

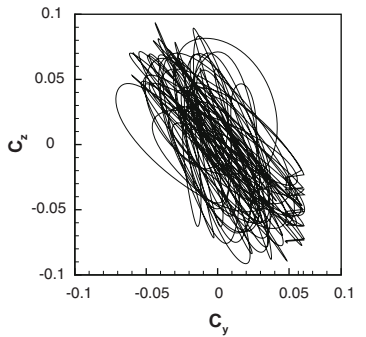

Fig. 8 Force diagrams for selected values of $R e$ on the route to chaos: $C_{x}-C_{y}$ diagram for $R e=220$ (a) and $R e=230$ (b); $C_{y}-C_{z}$ diagram for $R e=245(\mathbf{c})$ and $R e=270(\mathbf{d})$

threshold is characterised by the persistence of a reflectional symmetry plane and the occurrence of a second frequency. The corresponding attractor, depicted in Fig. 8a through its $C_{x}-C_{y}$ projection (for $R e=220$ ), is typical of a quasi-periodic system. The leading frequency $f_{0}$ is very close to the one existing in the previous regimes, while the secondary frequency $f_{1}$ is close to one-third of the leading frequency. ${ }^{1}$

For $R e=230$, the $C_{x}-C_{y}$ diagram (Fig. 8b) shows that the attractor becomes again a closed loop, indicating that the flow has come back to a periodic state. This can be explained as the result of a phase locking of the two main frequencies which, in this case, get into an exact 1:3 resonance. ${ }^{2}$ This exact resonance is lost when the Reynolds number is further increased and, for $R e=235$, the attractor (not shown) becomes again quasi-periodic and similar to the one plotted in Fig. 8a.

The planar symmetry is eventually lost for $R e \approx 240$. The last two plots (Fig. 8c, d) illustrate the shape of the attractor through its $C_{y}-C_{z}$ projection for $R e=245$ and $R e=270$, respectively. In the first case the trajectory seems to retain some symmetries as it does not explore the whole domain. This suggests that this regime is not yet completely chaotic but rather multi-periodic. On the other hand, in the second case a true chaotic state seems to be reached, as the trajectory makes larger excursions in the whole domain. We did not attempt to localise more precisely the threshold value of $R e$ associated with the onset of chaos, since computations are very time-consuming in this range of parameters. Moreover, a simple glance at the force diagrams is not sufficient to discriminate true chaos from multi-periodic solutions and more powerful means of investigation are required, such as the computation of Lyapunov exponents. Future work will be devoted to this issue.

\section{Summary}

In this paper, we used DNS to investigate the wake dynamics of a thick disk with an aspect ratio $\chi=3$, held fixed in an imposed upstream flow parallel to its symmetry axis. In the range $\operatorname{Re} \in[150,216]$, an original sequence of bifurcations was evidenced, which is somehow intermediate between the reference cases of a sphere and a thin disk, respectively. In the first two steps of the sequence, the flow successively encounters a steady, non-axisymmetric state $(S S)$ associated with a constant lift force, and a periodic, reflectional-symmetry preserving mode associated with a lift force oscillating about a non-zero mean value ("Zig-zig" mode). This sequence is identical to what happens in the wake of a sphere. However, a third bifurcation occurs, leading to an original type of flow which breaks the reflectional symmetry and oscillates in a quasi-periodic manner ("Knitknot" mode). Two additional bifurcations lead successively to a periodic, reflectional-symmetry-breaking mode ("Yin-Yang" mode) and to a periodic mode with reflectional symmetry and a lift force oscillating about zero ("Zig-Zag" mode). This part of the sequence is identical to what happens in the wake of a thin disk, except that in the latter case the "Yin-Yang" mode takes place after the second bifurcation of the sequence, immediately after the steady, non-axisymmetric state $(S S)$. In line with previous results obtained for the reference cases of a sphere and a thin disk, this whole sequence is fully explained by a theoretical model describing the interaction between the two leading modes. However, this model is unable to explain the dynamics encountered in the

\footnotetext{
${ }^{1}$ This nearly harmonic relation between the frequencies led us to call this regime a "Honky-Tonky" mode (as if it were a music instrument that would sound slightly out of tune).

${ }^{2}$ We thus propose to call this retuned mode a "Boogie-Woogie" mode.
} 
range $R e \in[217,270]$. In this range, at least three additional bifurcations exist, leading eventually to a fully chaotic state. Additional work is still required to completely describe this route to chaos.

This work is currently continued in the case where the objects are freely moving within the fluid under the effect of buoyancy and hydrodynamic forces. Preliminary results [2] have demonstrated an excellent agreement with the experiments conducted in our team [6,7]. They have also revealed the existence of several new kinds of trajectories, including steady oblique paths, periodic and quasi-periodic trajectories and weakly chaotic regimes. The cartography of these regimes is in progress. The application of normal form theory to this case is also a promising issue.

Acknowledgments The numerical simulations were performed on the Altix server computer of CICT in Toulouse, under grant P0727. The authors acknowledge Edgar Knobloch for fruitful discussions on the theoretical model.

\section{References}

1. Auguste, F., Fabre, D., Magnaudet, J.: Ecoulement de fluide visqueux autour d'un disque en incidence frontale. 18ème Congrès Français de Mécanique, Grenoble (France) (2007)

2. Auguste, F., Fabre, D., Magnaudet, J.: Numerical study of solid cylinders moving freely in a viscous fluid. EFMC7 Conference, Manchester (UK) (2008)

3. Bouchet, G., Mebarek, M., Ducek, J.: Hydrodynamic forces acting on a rigid fixed sphere in early transitional regimes. Eur. J. Mech. B 25, 321-336 (2006)

4. Crawford, J.D., Knobloch, E.: Symmetry and symmetry-breaking bifurcations in fluid dynamics. Ann. Rev. Fluid Mech. 23, 341-387 (1991)

5. Fabre, D., Auguste, F., Magnaudet, J.: Bifurcations and symmetry breaking in the wake of axisymmetric bodies. Phys. Fluids 20, 051702 (2008)

6. Fernandes, P.C., Risso, F., Ern, P., Magnaudet, J.: Oscillatory motion and wake instability of freely-rising axisymmetric bodies. J. Fluid Mech. 573, 479-502 (2007)

7. Fernandes, P.C., Ern, P., Risso, F., Magnaudet, J.: Dynamics of axisymmetric bodies rising along a zigzag path. J. Fluid Mech. 606, 209-223 (2007)

8. Golubitsky, M., Stewart, I., Schaeffer, D. M. (1988) Singularities and groups in bifurcation theory, vol. II. Applied Mathematical Sciences. Springer, Berlin

9. Johnson, T.A., Patel, V.C.: Flow past a sphere up to a Reynolds number of 300. J. Fluid Mech. 378, 19-70 (1999)

10. Magnaudet, J., Mougin, G.: Wake instability of a fixed spheroidal bubble. J. Fluid Mech. 572, 311-337 (2007)

11. Meliga, P., Sipp, D., Chomaz, J.M.: Unsteadiness in the wake of the sphere: receptivity and weakly non-linear global stability analysis. 5th BBVIV Conference, Costa do Sauipe (Brazil) (2007)

12. Mittal, R.: Planar symmetry in the unsteady wake of a sphere. AIAA J. 37, 388-390 (1999)

13. Mougin, G., Magnaudet, J.: Path instability of a rising bubble. Phys. Rev. Lett. 88, 14502 (2002)

14. Ormières, D., Provansal, M.: Vortex dynamics in the wake of a sphere. Phys. Rev. Lett. 83, 80-83 (1999)

15. Pesavento, U., Wang, Z.J.: Falling paper: Navier-Stokes solutions, model of fluid forces and center of mass elevation. Phys. Rev. Lett. 93, 14451 (2004)

16. Tomboulides, A.G., Orzag, S.A.: Numerical investigation of transitional and weak turbulent flow past a sphere. J. Fluid Mech. 416, 45-73 (2000)

17. Wilmarth, W.W., Hawk, N., Harvey, R.: Steady and unsteady motions and wakes of freely falling disks. Phys. Fluids 7, 197208 (1964) 